www.nature.com/jhg

\title{
EDITORIAL
}

\section{Special Section on Pharmacogenomics: recent advances and future directions}

Journal of Human Genetics (2013) 58, 305; doi:10.1038/jhg.2013.53

A $\mathrm{t}$ the Journal of Human Genetics, we are celebrating our first special section focusing on recent advances in pharmacogenomics: the study of the effect of genetic variations on responses to drugs.

During the past decade, human genetic research has made considerable progress, with advances in both genome analysis techniques and international research infrastructure for human genome information. In particular, genome-wide association studies have identified thousands of genetic variations that are associated with disease susceptibility and drug responses. These findings have improved our understanding of the mechanisms underlying disease pathogenesis and drug responses. In addition, this knowledge has revealed the genetic background that contributes to differences in disease susceptibility and drug responses between the individuals.

The next step is to translate the findings into clinical practice. Some of the identified genetic variations have large effects and are highly accurate at predicting drug responses. Therefore, such 'pharmacogenomic markers' are candidates for determining which patients will benefit from a particular drug. This special section contains eight articles on the current state of pharmacogenomic research and its implementation in the clinic.

Cancer pharmacogenomics is the focus of five reviews. Hertz and McLeod provide a broad outline of the influence of genomic variations on cancer treatment outcomes. They highlight examples of genetic variation that are predictors of cancer prognosis, response, toxicity and exposure. Ingle describes the pharmacogenomics of endocrine therapy in breast cancer. His review reveals the power of genome-wide association studies to identify key genes that affect drug responses, as well as the importance of functional studies for clarifying the underlying mechanisms of drug responses. The crucial scientific issues in the pharmacogenomic study of endocrine therapy for breast cancer are further discussed by Kiyotani et al., who focus on the controversial findings in studies assessing the effect of CYP2D6 variants on responses to the drug tamoxifen. In a research article, Yee et al. show the importance of pharmacogenomic research on hematological cancers and the difficulties entailed. In addition, Ishiguro, Yagi and Uyama of the Pharmaceuticals and Medical Devices Agency, Japan, review the usefulness and the limitations of pharmacogenomics-guided clinical trials for the regulatory approval of anti-cancer drugs in Japan.

Pharmacogenomic research on two major adverse drug reactions, severe cutaneous adverse reactions and drug-induced liver injury, is summarized by Kaniwa and Saito. This review highlights the important contributions of HLA alleles to these phenotypes.

Klein and Lee review the progress toward, and the challenges for, implementing clinical pharmacogenomic research on warfarin, a wellknown anti-coagulant. In addition, Perry and Shuldiner discuss the relationship between CYP2C19 variants and the pharmacokinetics and pharmacodynamics of, and clinical responses to, the newer anti-coagulant drug clopidogrel.

We sincerely thank the many authors, reviewers and editorial staff whose hard work and dedicated efforts have made this special issue possible. We hope that this exciting collection will be an enjoyable read and will help move personalized medicine one step closer to the clinic.

Taisei Mushiroda ${ }^{1}$, Kathleen M Giacomini ${ }^{2}$ and Michiaki Kubo ${ }^{1}$ ${ }^{1}$ Core for Genomic Medicine, Center for Integrative Medical Sciences, RIKEN Yokohama Institute, Yokohama, Japan and

${ }^{2}$ Department of Bioengineering and Therapeutic Sciences, University of California, San Francisco, CA, USA E-mail:mkubo@src.riken.jp 